\title{
Ignition of a nanosecond-pulsed near atmospheric pressure discharge in a narrow gap
}

\author{
Sarah Müller, Dirk Luggenhölscher and Uwe Czarnetzki \\ Institute for Plasma and Atomic Physics, Ruhr-University Bochum, 44780 Bochum, \\ Germany \\ E-mail: sarah.mueller@ep5.rub.de
}

\begin{abstract}
The ignition phase and the transition to quasi DC glow operation of a narrow-gap near atmospheric pressure discharge in hydrogen are investigated experimentally. The discharge is ignited by a short $10 \mathrm{~ns}$ voltage pulse with a peak voltage of $1.3 \mathrm{kV}$ followed by a $150 \mathrm{~ns}$ plateau of about $350 \mathrm{~V}$. Pulsing is at $12 \mathrm{kHz}$ which leaves a significant amount of residual charge between the individual pulses. Temporally resolved laser electric field measurement in the centre of the discharge employing a non-linear four-wave mixing scheme, ultra-high speed optical imaging by an ICCD camera at Balmer-alpha and Fulcher lines as well as the undispersed emission, and current and voltage measurements are performed. Special emphasis is put on a detailed analysis of the measured data by combining the results from the various diagnostics. This allows in addition to the directly measured quantities determination of the absolute evolution of the electron density, the development of space charge shielding, and the observation of the local electron dynamics. Pressure variations in a limited range indicate reasonable agreement with the Paschen law but raise also questions on the definition of the break down voltage under highly transient conditions.
\end{abstract}


Atmospheric pressure discharges under small spatial dimensions, so called microplasmas, have attracted large attention in recent years. On one hand large expectations on industrial applications exists while on the other hand the physics is often complex and difficult to investigate. In particular diagnostics is a challenge since most of the tools and techniques developed over decades for low-pressure, large scale discharges can not be applied here. Many types of microplasmas operate in pulsed mode and the transient phase of their ignition is even more difficult to access by adding to the already microscopic dimensions also fast temporal changes on ns or sub-ns timescales. Here an experimental investigation of the rapid ignition phase and the subsequent transition to quasi-DC operation in a molecular gas (hydrogen) is reported. The geometry is one-dimensional to allow a simple interpretation. The main parameters determined directly are the discharge voltage, the current density, the electric field in the centre of the discharge, and the spatially and temporally resolved emission. First successful application of these diagnostics to a similar setup was reported in [1-3]. Here we go beyond by more detailed studies and in particular a careful analysis and interpretation of the data. From the combination of the directly measured quantities with simple and robust models further insight in the ignition dynamics is provided. The temporal evolution of absolute electron densities is obtained and spatial and temporal development of different groups of low and highly energetic electrons is observed.

Here a discharge ignited in a plane geometry is investigated (figure 2) that allows a simple one-dimensional interpretation of the obtained results. Two parallel plate electrodes of $3 \mathrm{~cm}$ diameter and $1.2 \mathrm{~mm}$ gap width made from stainless steel form the discharge volume. The power coupling is performed by repetitive nanosecond highvoltage pulses with a rise time of $10 \mathrm{~ns}$, width of $\sim 10 \mathrm{~ns}$ and peak voltage of $1.3 \mathrm{kV}$. The high voltage pulse is followed by a quasi DC-phase of $350 \mathrm{~V}$ that lasts $150 \mathrm{~ns}$. The repetition rate is $12 \mathrm{kHz}$.

Short voltage pulses are generated from a DC power-supply by using a fast high-voltage switch (Behlke HTS-81). The discharge is operated between 200 mbar and 400 mbar of hydrogen. Simultaneous measurement of voltage and discharge current is performed by a high-voltage probe (Tektronix P6015A) and a current probe (Lecroy AP015) installed at the grounded electrode.

The electric field in the centre of the discharge is measured by a laser spectroscopic technique similar to CARS [4-6]. It is based on recording the intensity of infrared (IR) radiation generated through anti-Stokes Raman scattering on the gas molecules in the investigated medium. The excitation scheme used for the field measurement is presented in figure 1. The process can be described as four-wave mixing based on the third order non-linear susceptibility that leads to generation of coherent radiation with field dependent intensity: The first two waves $\omega_{1}$ and $\omega_{2}$ are provided by two lasers (intensities $I_{1}$ and $I_{2}$ respectively) which are colinearly superimposed and focused. The third wave involved in this process (with $\omega_{3}=0$ ) is effectively represented by the quasistatic electric field. The beam mixing leads to generation of a fourth wave $(2.4 \mu \mathrm{m})$ 


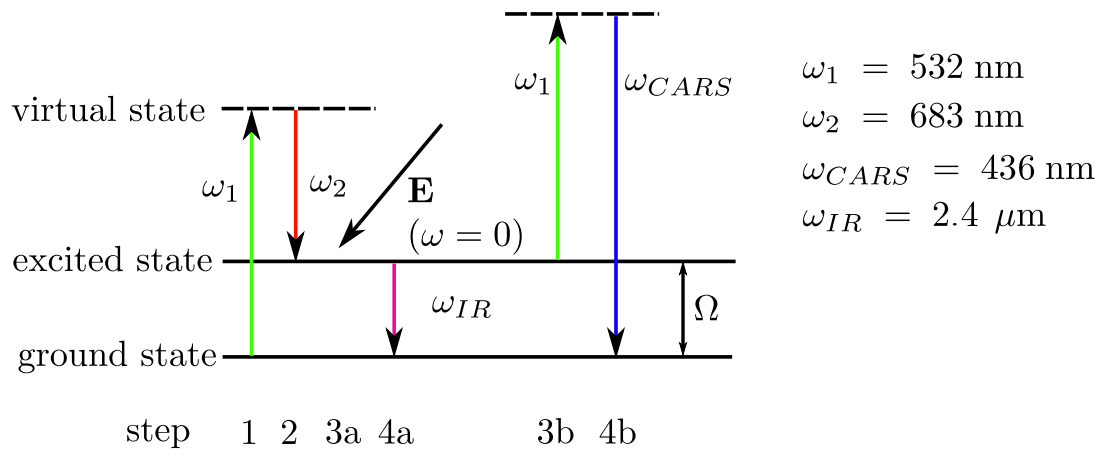

(a)

(b)

Figure 1. Excitation scheme relevant for CARS-based electric field measurement: (a) Generation of field-dependent IR-radiation. (b) Generation of field-independent anti-Stokes radiation.

whose intensity is proportional to the squared electric field strength. This scheme was originally invented by Ochkin et. al. [4-6]. Since the signal scales with the product of the green and red laser intensities the temporal resolution is slightly better than the individual laser pulse widths. We estimate a resolution (FWHM) of about $3 \mathrm{~ns}$. In addition to the field dependent signal $I_{I R}$, the anti-Stokes radiation ( $I_{C A R S}$ at $436 \mathrm{~nm}$ ) is detected. Both signals depend on the laser intensities and the population density difference between the involved states in the rotational-vibrational energy system:

$$
\begin{aligned}
& I_{I R}=\left(N_{\text {ground }}-N_{\text {excited }}\right)^{2} I_{1} I_{2}|E|^{2} \\
& I_{C A R S}=\left(N_{\text {ground }}-N_{\text {excited }}\right)^{2} I_{1}^{2} I_{2}
\end{aligned}
$$

By normalizing the static field dependent IR signal to the blue anti-Stokes signal, the dependence on the population distribution and the total gas density cancels out. Therefore, the electric field should linearly depend on the square root of the measured signal ratio:

$$
|E|=C \sqrt{I_{1} \frac{I_{I R}}{I_{C A R S}}}
$$

The calibration constant $C$ has to be determined experimentally. The laser intensity $\mathrm{I}_{1}$ remains in the dependency and can be recorded in parallel. In the experiment, this beam is provided by a solid state laser which has a rather stable output $( \pm 3 \%)$ so that $I_{1}$ is effectively a constant. In case of field inhomogenities along the propagation direction of the laser, the static field is the line average field. In any case, only the focal region contributes due to the non-linearity of the process. A schematic of the laser setup can be seen in figure 2. The laser system in this experiment consists of a frequency doubled injection seeded Nd:YAG laser at $532 \mathrm{~nm}$ (Continuum Powerlite Precision II) and a Nd:YAG-pumped dye laser at the Stokes wavelength $683 \mathrm{~nm}$ (Radiant Dyes Jaguar). Pulse energies used in the measurement are $30 \mathrm{~mJ}$ (Nd:YAG) and $5 \mathrm{~mJ}$ (Dye laser). Both laser beams are superposed linearly and focused by a plano-convex lens ( $\mathrm{f}=30$ 


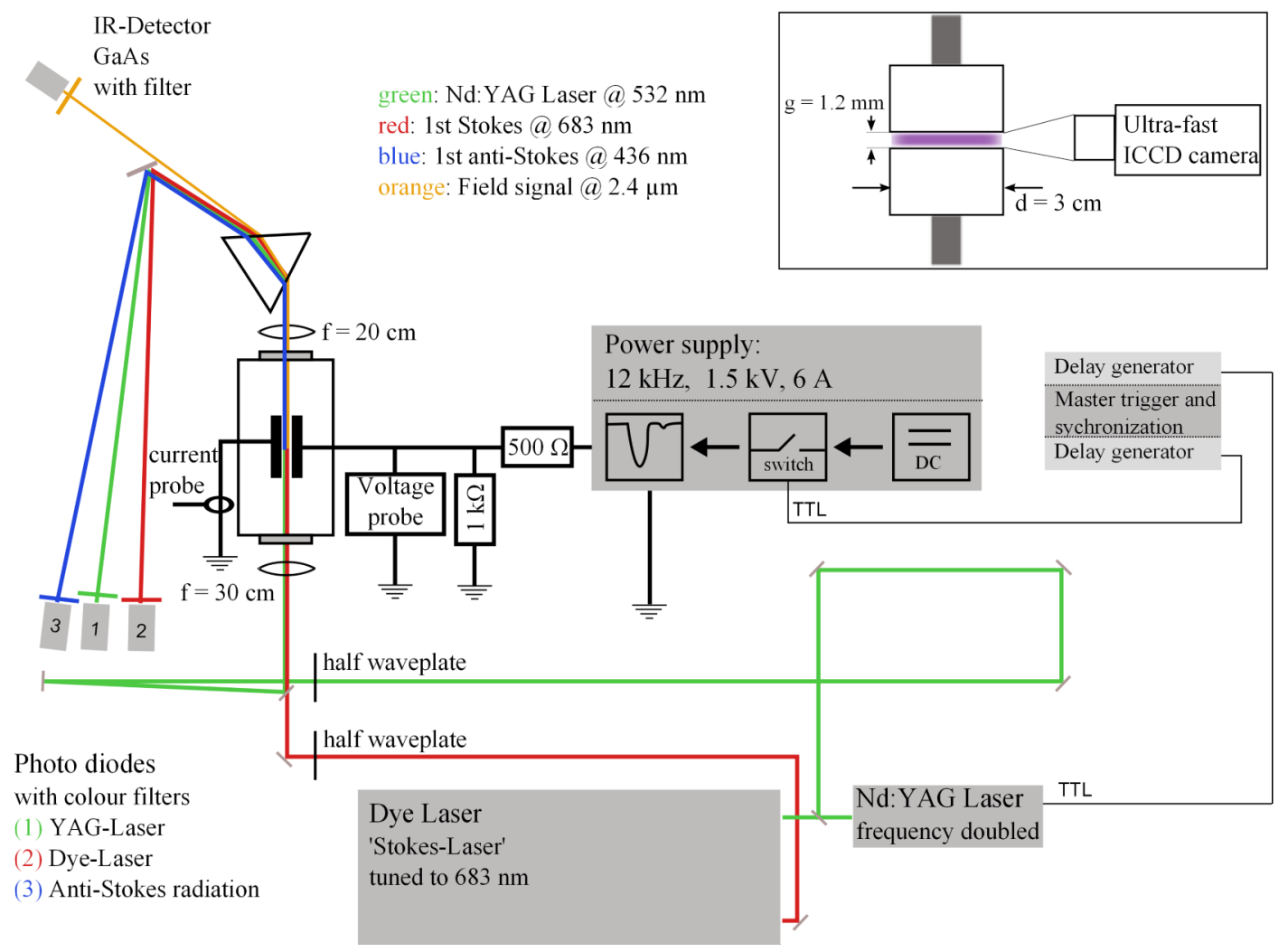

Figure 2. Experimental setup for the electric field measurements. Top right: optical setup for ICCD imaging.

$\mathrm{cm})$ into the the discharge. This results in a focal diameter of about $100 \mu \mathrm{m}$ that determines the spatial resolution along the discharge axis. The green laser radiation is aligned through a delay line to ensure equal optical beam paths and thus temporal overlay of both laser beams. The generated IR signal, the anti-Stokes radiation, and the laser beams are dispersed by a prism. The infrared radiation is focused on an IR detector (GaAs, Dorotek PVI-3TE) by a second plano-convex lens ( $\mathrm{f}=20 \mathrm{~cm}$ ). The remaining beam intensities are recorded by photo diodes (Thorlabs DET01A). Coloured filters in front of the three diodes detecting the visible radiation are used to separate the signal radiation from stray light originating from the optical setup. For the IR signal a $0.5 \mathrm{~mm}$ thick silicon wafer is used as a filter suppressing visible radiation.

The laser system is synchronized with the discharge circuit by a home-made frequency divider. The timing is controlled by delay generators (Stanford Research Systems DG535). Each measuring point during the discharge cycle represents an average of 50 laser shots over the signal intensities of generated radiation and the laser beams.

In order to obtain the calibration constant $C$, the intensities $\mathrm{I}_{1}, \mathrm{I}_{I R}$, and $\mathrm{I}_{C A R S}$ were detected while varying a static and homogeneous electric field without generation of a discharge. The calibration allowed also determination of the sensitivity. At 230 mbar, the minimum detectable field is $30 \mathrm{~V} / \mathrm{cm}$. With discharge operation the threshold is ten times higher due to electrical noise. 
The spatial-temporal emission at Balmer-alpha $\left(\mathrm{H}_{\alpha}\right)$, Fulcher lines, and the undispersed emission is detected by a fast ICCD camera (LaVision Picostar HR16). In this case quenching is beneficial since it reduces the lifetime of the excited states to values of the order of $10 \mathrm{ps}$ [7]. The ICCD imaging is synchronized with current, voltage and electric field measurements. A series of images with a step size of 250 ps was taken during the discharge evolution. The gate time was set to $0.5 \mathrm{~ns}$ and the accumulation time was $2 \mathrm{~s}$. Compared to the laser electric field measurement, the time resolution is much faster (0.5 ns vs. $3 \mathrm{~ns}$ ).

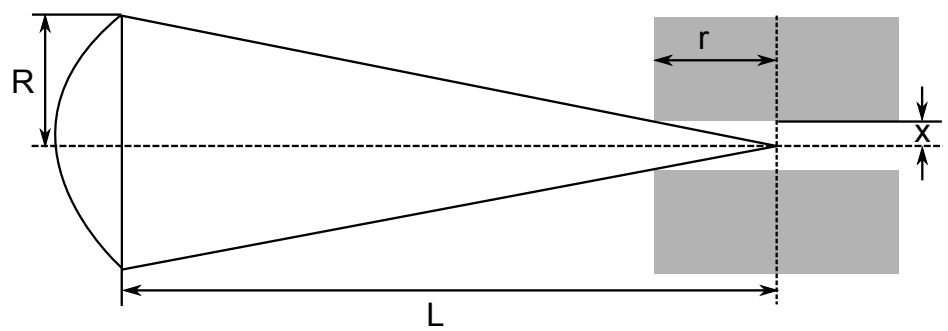

Figure 3. Shadowing of the observation angle occurs at the imaging of the discharge volume to the ICCD-chip. Here: $L=12 \mathrm{~cm}, r=1.5 \mathrm{~cm}, R=3 \mathrm{~cm}$.

Due to the setup geometry the observation cone is shadowed by the electrodes (figure 3). In order to compensate for the shadowing effect a dimensionless factor describing the relative depletion can be calculated [8]:

$$
B(y)=1-\frac{1}{\pi}\left(\arccos y-y \sqrt{1-y^{2}}\right)
$$

The variable $y=\frac{x \cdot L}{r \cdot R}$ is determined by the individual geometric dimensions. The factor $\mathrm{B}$ was taken into account in the image analysis.

The primarily measured data can be combined in a detailed analysis. In this analysis the mobility of electrons and ions is essential. Due to the high collisionality of the discharge, the ions are not able to move on the timescale of a few nanoseconds. The ion mobility $\mu_{\text {Ion }}=1.0 \cdot 10^{-3} \frac{\mathrm{m}^{2}}{\mathrm{Vs}_{\mathrm{s}}}[9]$ at $p=300$ mbar taking into account the maximal electric field value of $800 \mathrm{~V} / \mathrm{mm}$, the distance the ions are able to move is below $8 \mu \mathrm{m}$. Therefore, the ions can be considered to be fixed at the location of their generation. However, the electrons have a much larger mobility $\left(\mu_{e}=0.13 \frac{\mathrm{m}^{2}}{\mathrm{Vs}_{\mathrm{s}}}[10]\right)$. Under the same conditions as above, electrons therefore can transverse a distance of the order of the gap width. For the electron mobility the value reported by Raizer is used [10]. In the literature also other values are reported, e.g. [11], with differences in the absolute values of up to a factor of four. Further, the use of a constant value for the mobility is an approximation in itself and also field dependent mobilities are reported [12]. This uncertainty in the physical parameter sets limits to the absolute values derived in the subsequent data analysis. However, it should be noted that by the value used here at least consistency between different analysis approaches is achieved. 
Figure 4 shows temporally resolved measurements of voltage, discharge current and electric field. The applied voltage rises within $15 \mathrm{~ns}$ to the peak value of $1.3 \mathrm{kV}$. At the same time, the electric field strength reaches its maximal value $800 \mathrm{~V} / \mathrm{mm}$. A current peak $\left(I_{\max }=11 \mathrm{~A}\right)$ is measured 4 ns later.

The discharge cycle can be divided into two main phases: The ignition phase $(10<\mathrm{t}<$ $35 \mathrm{~ns}$ ) and a quasi-stationary phase (35 ns $<\mathrm{t}<220 \mathrm{~ns})$. When the voltage starts rising at 10 ns the electric field follows the applied voltage initially. At this early stage the space charge is still negligible but starts being developed. At 23 ns the gas breakdown occurs and the space charge starts shielding the applied electric field. During this time, the electric field is determined by the applied field reduced by the field produced by the total charge in the discharge volume. Assuming a homogeneous space charge, the reduced field can be expressed as

$$
E_{s c}(x, t)=\frac{V(t)}{d}+\frac{Q(t) x}{\epsilon_{0} d \cdot A}
$$

where $V(t)$ denotes the measured voltage, $A$ the discharge area (here: $7.1 \cdot 10^{-4} \mathrm{~m}^{2}$ ), and $d$ the gap width (here: $1.2 \mathrm{~mm}$ ). $x=\frac{d}{2}$ is the point of measurement. The charge $Q$ is determined by the integrated current:

$$
E_{s c}(t)=\frac{V(t)}{d}+\frac{1}{2 \epsilon_{0} A} \int_{0}^{t} I\left(t^{\prime}\right) d t^{\prime}
$$

This relation is represented by the early dotted line in figure 4 . The simplified relation for the space charge reduced electric field is valid until about 30 ns. The curve fits the measured data already very well, although it was calculated based on a homogeneous density throughout the discharge gap. Since the electrons are drawn to the anode, the development of a density gradient pointing in this direction can be expected. This can be taken into account tentatively by introduction of a weighting parameter in the second term of equation 6 . Assuming that $90 \%$ of the electrons are located between centre and anode, the calculated field decreases a few nanoseconds later than the dotted curve in figure 4 while the peak value deviates slightly more from the measured value. Overall, the difference is small.

The breakdown voltage can in principle be determined from these measurement. However, the transient nature of the fast ignition makes a clear definition difficult. In figure 5 the full symbols represent the voltage where the measured field starts to deviate from the nominal field. The open symbols are the voltage reached at the maximum field strength. Further, one might also extrapolate the steeply rising current to zero and determine the voltage reached at this time of sudden current increase as the breakdown value. This gives even higher voltages than shown in figure 5 . The results of figure 5 compare reasonably to the Paschen curve. As the secondary emission coefficient $\gamma$ is not known exactly two curves representing the lower and upper limits of the probable parameter range are shown $(\gamma=0.1$ and $\gamma=1)$.

Townsend's ionization coefficient $\alpha$ provides a convenient means to estimate the electron density increase out of the measured electric field strength. Neglecting spatial 


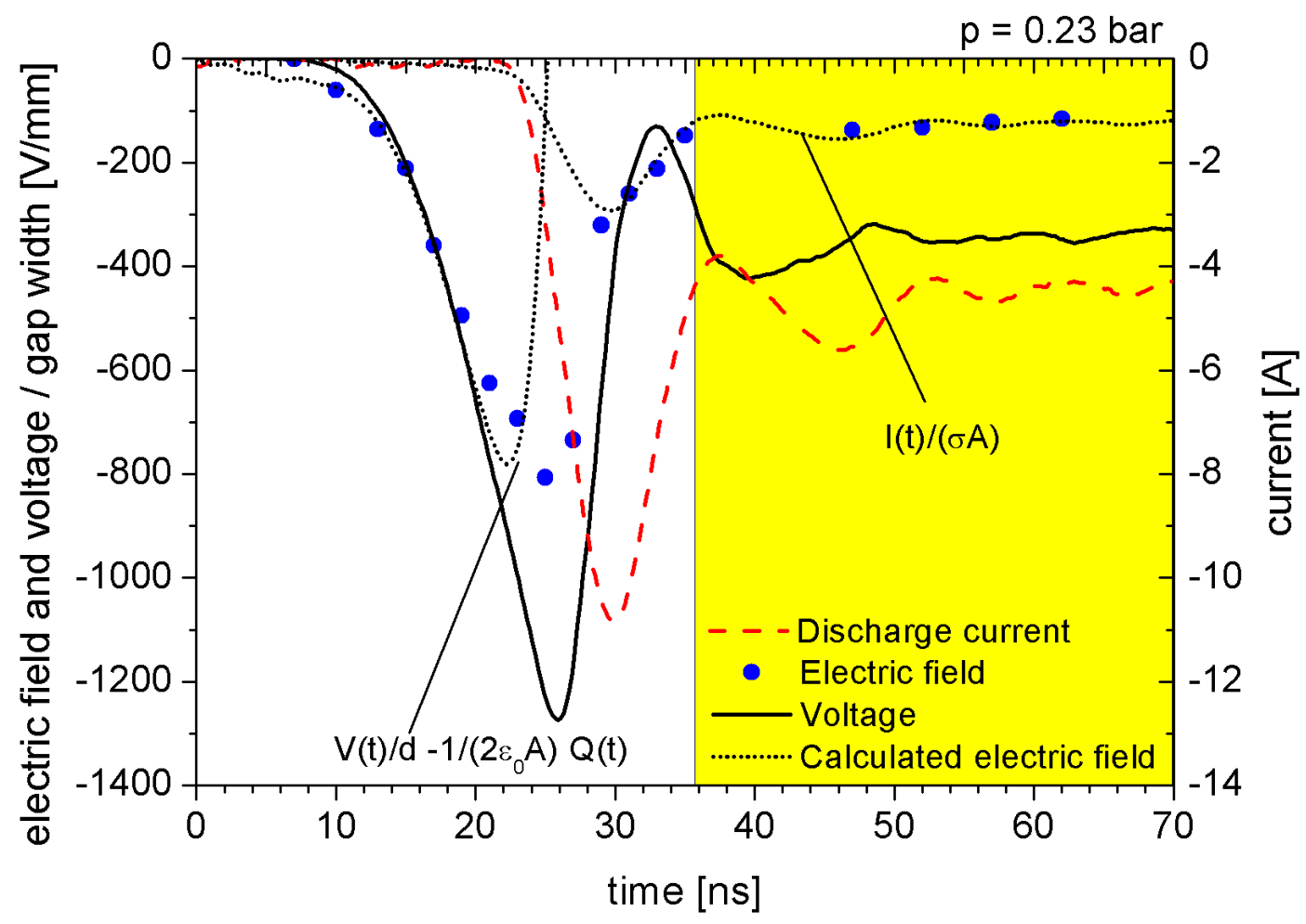

Figure 4. Temporally resolved measurement of voltage, discharge current and electric field. The dotted curve shows the calculated electric field. The coloring indicates the beginning of the quasi-stationary phase of the discharge evolution at about $35 \mathrm{~ns}$, while the ignition phase is uncoloured.

gradients of the flux in the continuity equation, the local density becomes:

$$
\begin{aligned}
& \frac{\partial}{\partial t} n=\alpha \mu_{e} n E \\
& \Rightarrow n=n_{0} \exp \left\{\mu_{e} \int_{0}^{t} \alpha(E) E d t^{\prime}\right\} .
\end{aligned}
$$

The first Townsend coefficient is given by the empirical formular $\alpha=A p \exp \left\{-\frac{B p}{E}\right\}[10]$. The ionization coefficient together with the gas dependent constants $\mathrm{A}$ and $\mathrm{B}$ are taken from [10], $A p=846.7 \mathrm{~cm}^{-1}$ and $B p=22481.2 \mathrm{Vcm}^{-1}$ at $230 \mathrm{mbar}$.

A density increase by factor of about 66 is determined (black diamonds in figure 6 ). The electron density rises rapidly in parallel with the measured current within an interval of only $8 \mathrm{~ns}$. Subsequently, the density stays constant since the lower electric field strength is no longer sufficient for a significant ionization rate.

The diffusion time in the afterglow is about 100 times longer than the time between successive pulses of $\tau=8.3 \cdot 10^{-5} \mathrm{~s}$. Since diffusion can therefore be neglected, the plasma is decaying by recombination. Due to the large cross section, initially produced $\mathrm{H}_{2}^{+}$-ions are immediately converted to $\mathrm{H}_{3}^{+}$[13-15]. The recombination rate of $\mathrm{H}_{3}^{+}$with electrons is $k=2 \cdot 10^{-7} \mathrm{~cm}^{3} / s$ [16]. The initial density is then independent of the final 


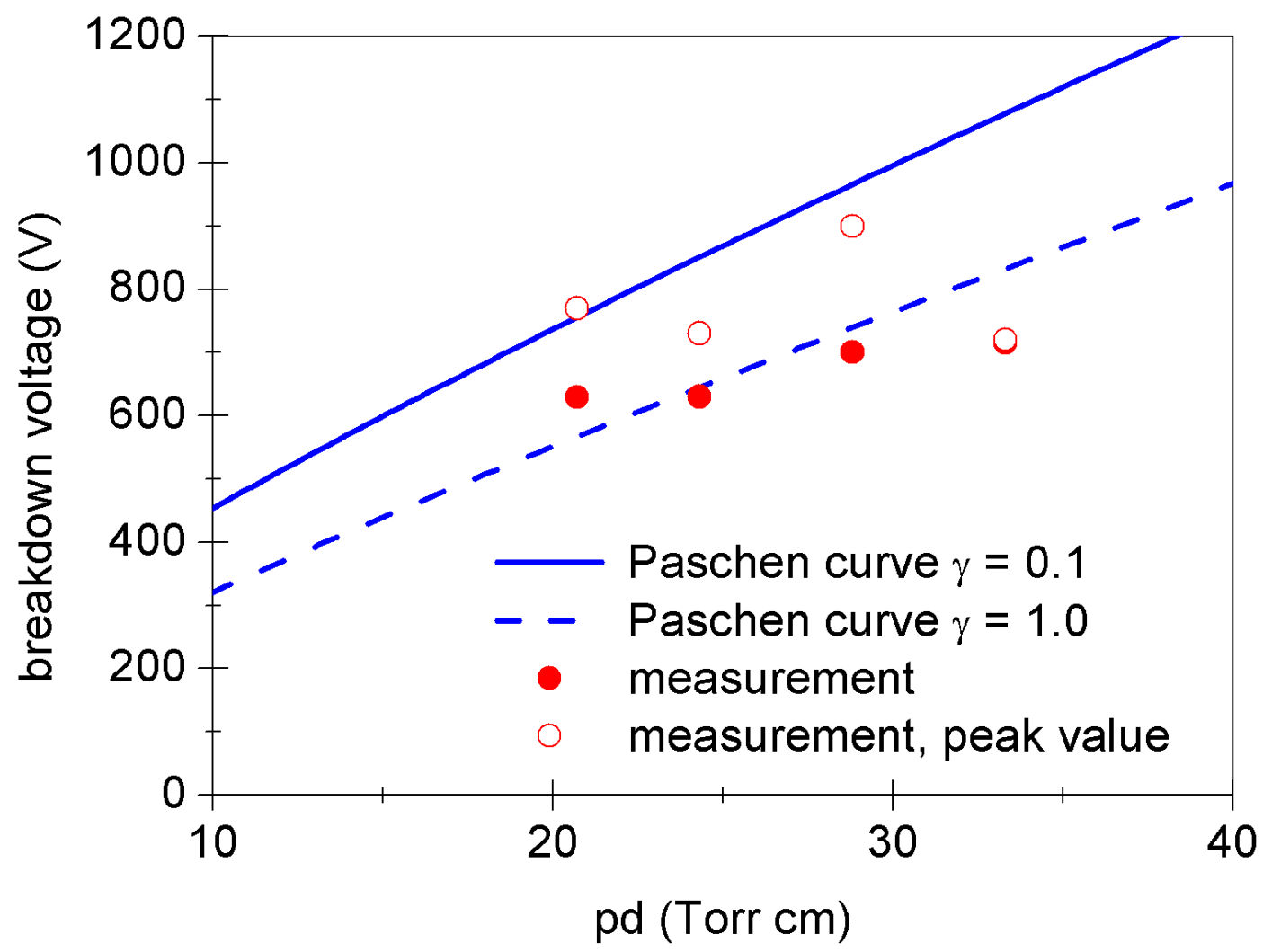

Figure 5. Breakdown voltage resulting from the field measurements. Full symbols denoted the reading when the electric fields starts to deviate from the applied voltage, the open symbols result from the measured peak values.

density at the end of the discharge cycle and given by $n=1 / k \tau=3 \cdot 10^{16} \mathrm{~m}^{-3}$. Based on this initial value the final density becomes $4.0 \cdot 10^{18} \mathrm{~m}^{-3}$ in the quasi-static phase.

The absolute value of the plasma density can also be derived time resolved from the measured current and electric field strength. In this context it is important that ions do not move and charges are not lost by diffusion or recombination on the ns-timescale. The total current in the centre of the discharge is:

$$
I=A \cdot j=A\left(\sigma E+\epsilon_{0} \dot{E}\right)
$$

Here the first term represents the conduction current with the conductivity $\sigma$ and the second term the displacement current. Since the conductivity $\sigma$ is density dependent, an expression providing the electron density time resolved during the whole discharge cycle can be found:

$$
n(t)=\frac{1}{E(t) e \mu_{e}}\left(\frac{I(t)}{A}-\epsilon_{0} \dot{E}(t)\right)
$$

The initial density determined in this way is $4.5 \cdot 10^{16} \mathrm{~m}^{-3}$ (red squares in figure 6). This value compares remarkably well with the value estimated from the recombination. Also the rapid density development and transition to a quasi stationary value compares well to the analysis above based on the Townsend coefficient. The quasi stationary density 


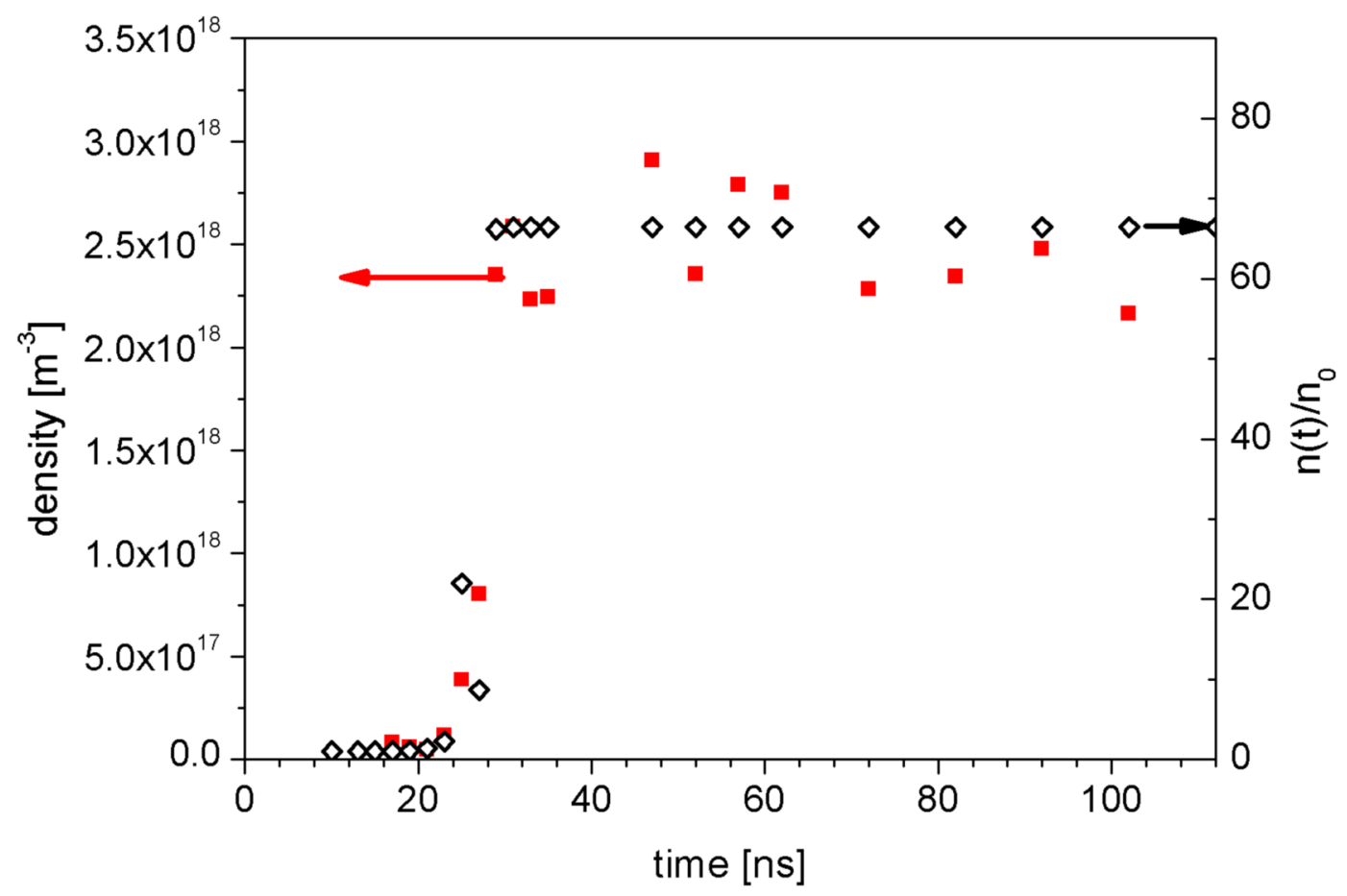

Figure 6. Black diamonds and right axis: density increase during the ignition process calculated from the field dependent Townsend ionization rate (equation 8). Red squares and left axis: Time evolution of the electron density derived from the current and electric field measurement (equation 10).

Scaling of the right $y$-axis was selected to match the absolute values on the left axis.

is $2.5 \cdot 10^{18} \mathrm{~m}^{-3}$, corresponding to an increase by a factor of 56 which is very close to the factor 66 derived above.

The constant density established in the quasi dc-phase is equivalent to a constant conductivity. In this phase, the electric field varies only slowly and the displacement current is negligible. Therefore, a direct proportionality between the current and the electric field exists. This proportionality is represented in figure 4 by the second dotted curve which connects the measured electric field data at later times. Excellent agreement is found throughout. At times earlier than $30 \mathrm{~ns}$, the electric field varies rapidly, the displacement current becomes dominant, and the simple proportionality breaks down. Detailed insight into electron dynamics is provided by high-speed ICCDimaging. Spatially and temporally resolved measurement of visible radiation emitted by the discharge supports the time-resolved field measurements and their interpretation. In figure 7 the spatio-temporal evolution of the emission is shown. The imaging was performed unfiltered (figure $7 \mathrm{a}$ ) and using interference filter to solely transmit $H_{\alpha}$-line (figure 7c) or the hydrogen Fulcher bands (figure 7b).

In agreement with current, voltage, and field measurements the ignition (23 ns - $35 \mathrm{~ns}$ ) and subsequent quasi DC-phase can be identified. In addition, an early (17 ns - $23 \mathrm{~ns}$ ), probably Townsend, regime becomes visible by very weak emission from the Fulcher lines 
(7b) and the undispersed measurement (7a) at the very early time of voltage rise. Here, an initial electron density remaining from the preceding discharge cycle is present and distributed probably homogeneously over the electrode gap since recombination leads to homogenization of the density distribution. At $\mathrm{t}=10 \mathrm{~ns}$, the voltage starts rising and the resulting electric field attracts the electrons to the anode. The pre-breakdown phase (17 ns - $23 \mathrm{ns)}$ is characterized by a relatively weak emission maximum stretching from $0.4 \mathrm{~mm}$ distance from the cathode to the anode (figure $7 \mathrm{a}$ ). The electron density is still very low at this early stage. The dark region in front of the cathode can be interpreted as an ion space charge from where electrons have been removed. The electron drift distance can be calculated from the measured field by integrating the drift velocity $u$ in time. Here it is important that space charge shielding is not yet effective and the field is homogeneous. With $u=\mu_{e} \cdot E$ it follows:

$$
s=\mu_{e} \int_{10 n s}^{23} E d t^{\prime}
$$

The result $s=0.4 \mathrm{~mm}$ matches the value found in the emission measurement very well. Since no atomic hydrogen is produced yet, emission in the $H_{\alpha}$-region is absent. With increasing field also ionization and electron density are rising. While the electron density increases by only a factor of about 60 , the emission increases by more than three orders of magnitude in the ignition phase. Light emission starts to rise over the whole gap simultaneously. Since the voltage keeps rising, the breakdown condition is reached soon $(\mathrm{t}=26 \mathrm{~ns})$. At this time a very rapid ionization wave proceeds from anode to cathode [17]. The tilting of the ionization front is hardly visible in the overview graph shown in figure 7. A zoom into the ignition interval is, therefore, provided in figure 8. Its propagation velocity can be roughly determined to $1 \cdot 10^{6} \mathrm{~m} / \mathrm{s}$. In parallel to the wave propagation a cathode sheath is developed. The wave slows down when approaching the edge of the sheath at a distance of $200 \mu \mathrm{m}$ from the cathode. The $\mathrm{H}_{\alpha}$ emission intensity at this time peaks in the sheath with an amplitude of almost one order of magnitude higher than in the ionization wave. This is probably caused by massive secondary electron emission from the cathode. These secondary electrons will then gain much higher energies than the electrons in the bulk. Indeed, the emission at the Fulcher lines does not show the peak found at $\mathrm{H}_{\alpha}$. This is consistent since the cross sections have a quite distinct energy dependence [18-20]. While the atomic cross section has a late maximum at about $60 \mathrm{eV}$ with a very slow decay at higher energies, the molecular cross section peaks already at $15 \mathrm{eV}$ and decays quickly at higher energies. Consistently the Fulcher lines are more efficiently excited in the bulk when low electron energies are expected.

After the ionization wave has traveled to the cathode, the current keeps rising and produces a second emission maximum around $30 \mathrm{~ns}$. Subsequently, together with the decreasing current, the emission intensity decreases to an absolute minimum $(\mathrm{t}=40$ ns). A sheath region in front of the cathode and a bulk region with less intense emission is generated. The emission in the quasi dc-phase is characterized by excitation via 


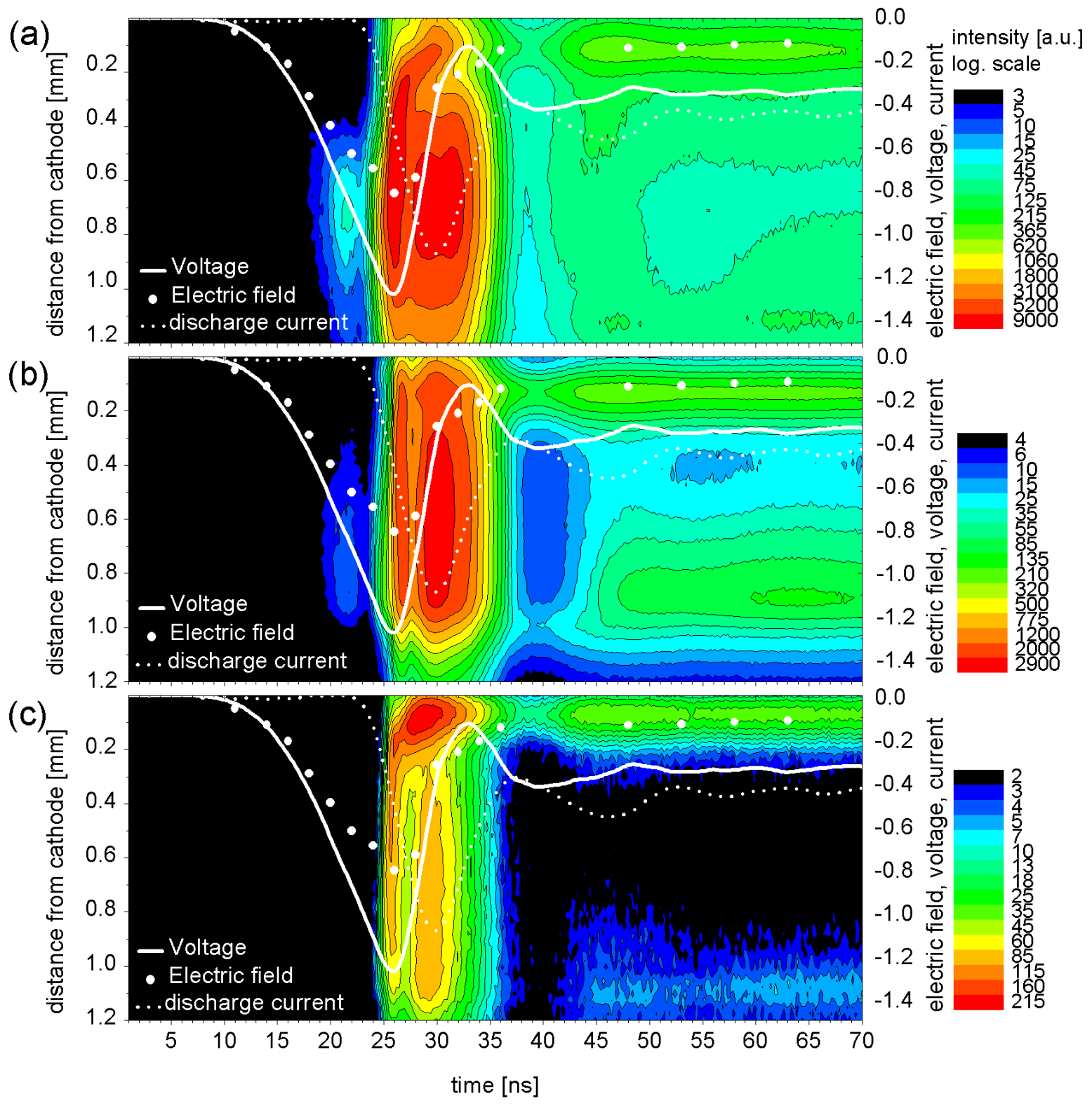

Figure 7. Spatially and temporally resolved and radially integrated measurement of visible radiation emitted by a nanosecond pulsed discharge and simultaneous measurement of electric field $[\mathrm{kV} / \mathrm{mm}]$, applied voltage $[\mathrm{kV}]$, and plasma current $[10$ A]. (a) Unfiltered emission. (b) With interference filter transmitting between $600 \mathrm{~nm}$ and $625 \mathrm{~nm}$ (Fulcher-series). (c) $\mathrm{H}_{\alpha}$-line recorded with interference filter.

secondary electrons generated in the cathode sheath. Some weaker emission can also be identified close to the anode. The major part of the bulk is free of Balmer-alpha emission. Since it is unlikely that the atomic hydrogen generated in the ignition phase has vanished only nanoseconds later, this indicates the absence of energetic electrons. The Fulcher line sensitive to low energetic electrons still show some emission in this region with a peak also close to the anode.

By considering the sheath edge to be located at the point of maximal emission, the 


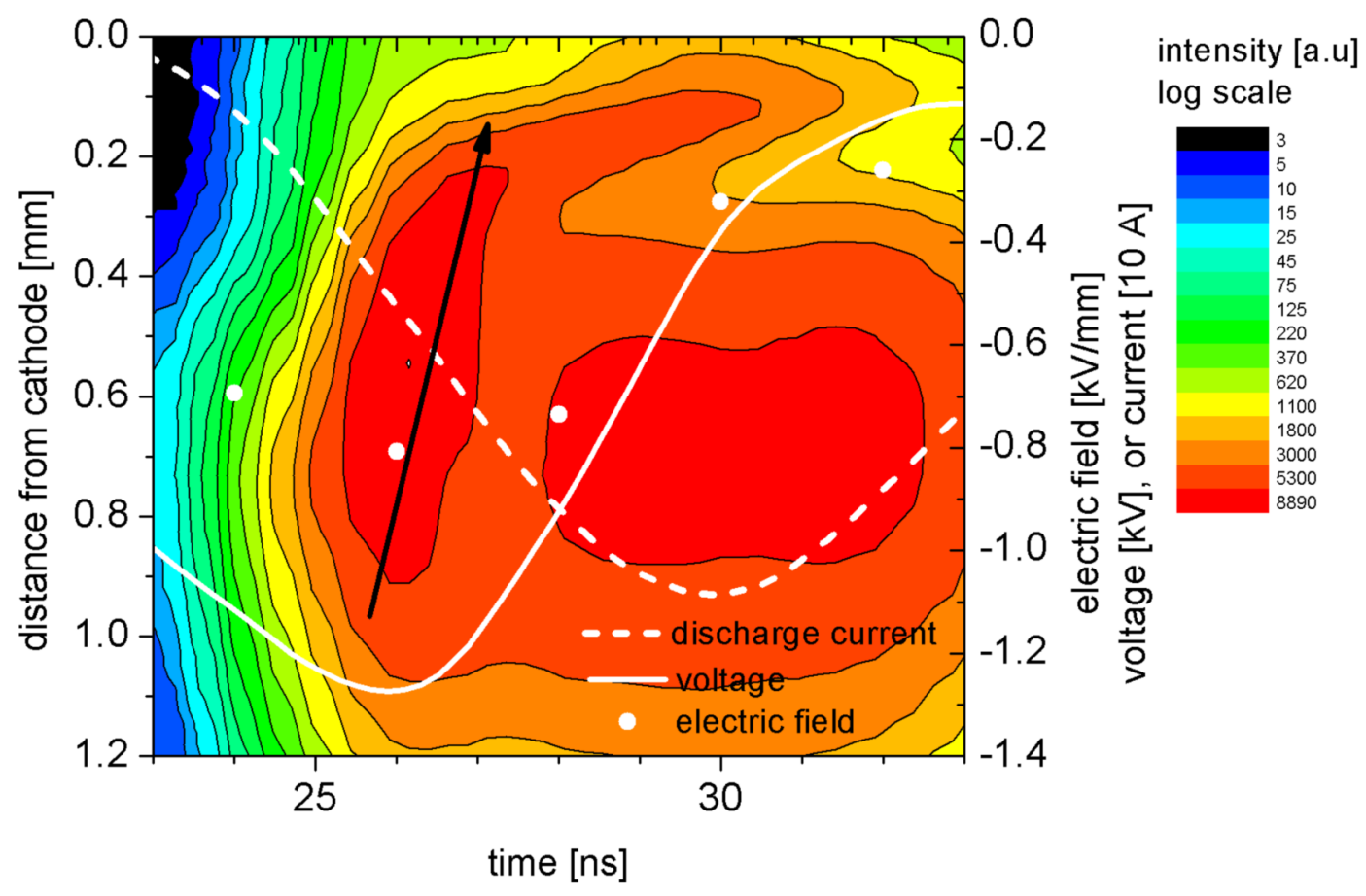

Figure 8. Zoom into the ignition phase. Example here: Spectrally averaged measurement. The arrow indicates the ionization wave traveling from the anode to the cathode with a velocity of about $10^{6} \mathrm{~m} / \mathrm{s}$.

sheath thickness can be determined to be $100 \mu \mathrm{m}$ in the $\mathrm{H}_{\alpha}$, and $125 \mu \mathrm{m}$ in the Fulcher, and undispersed emission. The larger value is probably the more reliable one since in the Fulcher and the spectrally averaged measurement are more sensitive to lower energetic electrons.

The density in the sheath can be estimated from the sheath width and the voltage balance across the gap $U_{\text {total }}=U_{s}+U_{\text {bulk }}$. If the field in the bulk is assumed to be homogeneous, the measured electric field gives a bulk voltage drop of $163 \mathrm{~V}$. With the total voltage $350 \mathrm{~V}$, the sheath voltage can be determined to $187 \mathrm{~V}$. Assuming a homogeneous density inside the sheath (homogeneous ionization and a negligible ion drift on this time scale) the density in the sheath can be calculated from Poisson's equation using the sheath width $s=125 \mu \mathrm{m}$

$$
n=\frac{2 U_{s} \epsilon_{0}}{e s^{2}} .
$$

A sheath density $n=1.3 \cdot 10^{18} \mathrm{~m}^{-3}$ results from this equation. This value is by a factor two smaller than the value determined in the bulk by evaluation of the current and field measurement, which seems to be reasonable.

The discharge parameters in the quasi DC-phase can be compared to the tabulated literature values for normal DC discharges [10]. This gives a sheath voltage of $250 \mathrm{~V}$ (here $190 \mathrm{~V}$ ), a current of $15 \mathrm{~A}$ (here $5 \mathrm{~A}$ ) and sheath width of $50 \mu \mathrm{m}$ (here $125 \mu \mathrm{m}$ ). Although certain deviations are obvious, the order of magnitude values are very close. 
In conclusion the measured data provide a detailed insight into the discharge and ignition dynamics. By applying some simple but robust analysis procedures further plasma parameters are inferred. In particular the temporal evolution of the absolute plasma density is revealed.

These measured quantities and the parameters derived by the analysis procedure should provide a useful benchmark data set for simulation of discharge ignition at near atmospheric pressures.

\section{Acknowledgments}

This work was supported by the 'German Research Foundation' (DFG) in the frame of 'Research Unit 1123 - Physics of Microplasmas', the Research Department 'Plasmas with Complex Interactions' of the Ruhr-University Bochum, and the Research School of the Ruhr-University Bochum.

The authors gratefully thank Igor Adamovich, Zoltan Donko, Julian Schulze, and Satoshi Hamaguchi for fruitful discussions.

\section{References}

[1] T. Ito, K. Kobayashi, S. Müller, D. Luggenhölscher, U. Czarnetzki and S. Hamaguchi $2009 \mathrm{~J}$. Phys. D: Appl. Phys. 42092003

[2] T. Ito, K. Kobayashi, U. Czarnetzki and S. Hamaguchi 2010 J. Phys. D: Appl. Phys. 43062001

[3] S. Müller, T. Ito, K. Kobayashi, D. Luggenhölscher, U. Czarnetzki and S. Hamaguchi $2009 \mathrm{~J}$. Phys.: Conf. Ser. 227, 012040

[4] O. A. Evsin, E. B. Kupryanova, V. N. Ochkin, S. Y. Savinov and S. N. Tskhai 1995 Quantum Electronics 25278

[5] A. M. Zheltikov, N. I. Korotev, A. N. Maumov, V. N. Ochkin, S. Y. Savinov and S. N. Tskhai 1999 Quantum Electronics 2973

[6] V. N. Ochkin and S. N. Tskhai 2002 Physics-Uspekhi 461214

[7] A. Francis, U. Czarnetzki, H. F. Döbele and N. Sadeghi 1997Appl. Phys. Letters 7129

[8] B. L. Preppernau and T. A. Miller Glow discharge Spectroscopy (New York: Plenum NY)

[9] E. W. MacDaniel and E. A. Mason 1973 The mobility and diffusion of ions in gases New York: John Wiley and Sons (1973)

[10] Y. Raizer (1991) Gas Discharge Physics (Heidelberg: Springer)

[11] S. J. Buckman and A. V. Phelps 1985 J. Chem. Phys. 82, 4999,

[12] A. E. D. Heylen 1960 Research Notes

[13] T. Simko, V. Martisovits, J. Bretagne and G. Gousset 1997 Phys. Rev. E 56, 5908

[14] A. V. Phelps 1990 J. Phys. Chem. Ref. Data 19, 653

[15] U. Czarnetzki, D. Luggenhölscher, and H. F. Döbele 2001 Appl. Phys. A 72509

[16] R. Johnsen 2005 J. Phys.: Conference Series 483

[17] A. N. Lagarkov, I. M. Rutkevich 1994 Ionization waves in electrical breakdown of gases (New York: Springer)

[18] R. K. Janev, W. D. Langer, K. Evans and D. E. Post Elementary processes in hydrogen helium plasmas, Springer 1987

[19] G.R. Möhlmann, FJ. de Heer and J. Los 1977 Chemical Physics 25 103-116

[20] G.R. Möhlmann and FJ. de Heer 1976 Chem. Phys. Letters 43 (1976) 240-245 